\title{
Review
}

\section{Dietary patterns, caloric restrictions for management of cardiovascular disease and cancer; a brief review}

\author{
Heidi Bochenek ${ }^{1}$, Irena Krga ${ }^{2}$, Domenico Sergi ${ }^{3}$, Matina Kouvari ${ }^{1,4,5, *}$, Manja Zec ${ }^{2,6}$, \\ Nenad Naumovski ${ }^{1,4,5, *}$ \\ ${ }^{1}$ Functional Foods and Nutrition Research (FFNR) Laboratory, University of Canberra, ACT 2617 Bruce, Australia \\ ${ }^{2}$ Institute for Medical Research, National Institute of Republic of Serbia, University of Belgrade, 11000 Belgrade, Serbia \\ ${ }^{3}$ Department of Translational Medicine, University of Ferrara, 44121 Ferrara, Italy \\ ${ }^{4}$ Department of Nutrition and Dietetics, School of Health Science and Education, Harokopio University, 10431 Athens, Greece \\ ${ }^{5}$ Discipline of Nutrition and Dietetics, Faculty of Health, University of Canberra, ACT 2601 Canberra, Australia \\ ${ }^{6}$ School of Nutritional Sciences and Wellness, University of Arizona, Arizona, Tucson, AZ 85721, USA \\ *Correspondence: Nenad.naumovski@canberra.edu.au (Nenad Naumovski); matina.kouvari@canberra.edu.au (Matina Kouvari) \\ Academic Editor: Samy I. McFarlane \\ Submitted: 15 November 2021 Revised: 17 December 2021 Accepted: 27 December 2021 Published: 20 January 2022
}

\begin{abstract}
Cardiovascular disease (CVD) and cancers are overall still identified as the two most prevalent non-communicable diseases globally. Their prevention and potential reversal (in particular CVD risk) was seen effective with the modification of dietary intake that was applied in several different populations. Although the findings from epidemiological studies provide support that adhering to dietary patterns such as the Mediterranean diet can reduce incidence and prevalence of CVD and some forms of cancer, the mechanistic aspects of disease modulation associated with both diseases can be seen in dietary management. Several studies have already explored the potential modes of action of certain nutrients in well controlled large clinical trials. However, the clinical trials designed to determine the effects of adhering to a particular diet are relatively hard to conduct and these studies are faced with several obstacles particularly in the populations that are identified with a high risk of CVD or different cancers. Therefore, it is important to understand potential underlying and shared mechanisms of action and to explore how healthy dietary patterns may modulate the occurrence, initiation, and progression of such diseases. The aim of this review is to summarise and conceptualize the current understanding relating to healthy dietary patterns, and briefly discuss the opportunities that epigenetic research may bring and how it may assist to further interpret epidemiological and clinical evidence.
\end{abstract}

Keywords: Cardiovascular disease; Cancer; Dietary patterns; Nutrition; Caloric restrictions

\section{Introduction}

Cardiovascular disease (CVD) and cancer overall represent the two most prevalent non-communicable diseases globally [1]. Furthermore, these two diseases also contribute to the major causes of mortality worldwide, with rates of over $70 \%$ [1]. The onset, progression and sustenance of both diseases share very similar underlying risk factors and potential mechanisms including obesity, lowgrade chronic inflammation and metabolic derangements $[1,2]$. As many as $90 \%$ of cancer cases and approximately $80 \%$ of cardiometabolic diseases including CVD, may be attributed to modifiable risk factors such as dietary choices and physical activity. Therefore, interventions through modulation of lifestyle-related factors such as diet, may be an attractive and effective option for their prevention and onset [1]. To this issue, it is estimated that among all behaviours, nutrition makes the largest contribution to CVD morbidity across Europe [3]. Similarly, the estimation by the World Cancer Research Fund reports that anywhere between 3-4 million cancer cases globally could have been avoided through a healthier lifestyle while adhering to a healthy dietary pattern can contribute to the prevention of cancer by up to $30 \%$ [4]. It is well established that diet may influence several facets of these two diseases, including initiation, progression, quality of life, treatment outcomes and survivorship.

The impact of diet on CVD and cancer has evolved substantially over the past few decades, initially relying on the findings of epidemiological and symptomatic evidence, to current methods of evaluating biomarkers to quantify dietary impact on health and disease [5-7]. The adherences to several healthy dietary patterns and non-communicable diseases outcomes are well reported in observational studies [8-12]. Among these, adherence to the Mediterranean diet (MedD) has been extensively studied in primary CVD prevention in healthy individuals and in individuals at an increased cardiometabolic risk [10,11]. The results from these studies have been particularly promising, with positive outcomes reported for participants with different stages of CVD [10,12-15]. Similarly, results of a relatively recent meta-analysis indicate that healthy and predominately plant-based dietary patterns (in comparison to Western- 
style dietary patterns) may decrease the risk of various types of cancer (i.e., colorectal) [16]. In addition to high-quality dietary pattern adherences, recent discussions have also arisen regarding the role of hypocaloric diets (i.e., calorie restriction) and further novelties in nutrition epidemiology implying the benefits of fasting on potential suppressions in onset of both CVD and cancer.

Therefore, the aim of this brief narrative review is to collate the latest evidence and discuss the role of diet on two major non-communicable diseases, namely CVD and cancer. Furthermore, we aim to present the evidence from the hitherto literature regarding isocaloric healthy dietary patterns as well as typical hypocaloric diets or diets that follow the scheme of intermittent fasting.

\section{Shared impacts of diet on cardiovascular disease and cancer progression}

The global prevalence of adolescent obesity has doubled since the 1980 's, with over $23 \%$ of the global adolescent population considered obese [17]. Furthermore, very few cases occur as a result of syndromic obesity and the most significant cause of this increase is mainly due to modifiable factors such as societal alterations in food (high energy density, food processing and food availability) contributing to over-nutrition [17]. In addition, obese adolescents will continue to be obese in their adult years contributing further to the development, progression and sustenance of cardiometabolic diseases and various forms of cancer. Therefore, the optimal time to instil dietary and lifestyle changes to prevent development of cardiometabolic disease is between the ages of 7 and the onset of puberty [17].

Similar patterns were also observed in cancer development. A multiethnic study including 2.3 million adolescent participants and a 45 year follow up period indicated a strong link between increased body mass index (BMI) and cancer development $[17,18]$. The findings of the study indicate that high BMI (over 35) in late adolescence was strongly associated with the development of haematological and solid malignancies such as pancreatic cancer, colorectal cancer, gastroesophageal adenocarcinoma and renal cell carcinomas in adulthood, regardless of ethnicity [17-19]. Therefore, interventions targeting body weight reductions (primarily focused on the reduction of body fat) at an early age may prevent occurrence of future health complications. However, no interventional strategies have thus far been effective at decreasing obesity rates or continued obesity from adolescence to adulthood [17-19].

From the pathophysiological perspective, obesity may induce chronic oxidative stress and low-grade chronic inflammation. This metabolic inflammation is mainly driven by lipotoxicity and adiposiopathy associated with dietary pattern, dietary triggers and visceral adipose tissue deposits. Furthermore, this has to be seen as a two-way approach as adipose tissue is also an endocrine organ able to secrete pro-inflammatory factors that are more seen in the visceral fat [20,21]. Several pro-inflammatory factors secreted by adipocytes include adipocytokines, cytokines, tumour necrosis factor-alpha (TNF- $\alpha$ ) and a number of interleukins (IL) contributing to the increased inflammation itself. Therefore, the presence of inflammation further promotes the secretion of pro-inflammatory factors contributing to the continuous inflammatory cycle itself $[1,2,22]$. The increased presence of these inflammatory factors alone and onset of inflammatory cycling is implicated in the development and pathogenesis of several cancers and onset of CVD [20].

In addition, the Warburg effect is most notably associated with cancer, being the driving force behind two hallmarks of cancer; uncontrolled cellular hyper-proliferation and reprogramming of energy metabolism [22]. While the prevalence of the Warburg effect is less described in CVD, it nevertheless plays an important role as a contributing factor to the progression of CVD [23]. It is proposed that imbalance caused by metabolic changes may subsequently cause cardiac dysfunction and impairment in the form of cardiac hypertrophy $[23,24]$. These complications are further compacted by the endogenous and pathological production of fructose by injured cardiac myocytes, most often following coronary heart disease $[23,24]$. While the Warburg effect can be observed in both CVD and cancer separately, it is evident that the effect may act as a potential metaphorical bridge that links both diseases directly.

\section{Importance of dietary patterns over single nutrients}

As previously described, cancer and CVD are particularly influenced by modifiable lifestyle factors, of which diet represents a key discriminant in shaping human health trajectories. Indeed, diet can both exert deleterious as well as protective effect on health, depending on a plethora of factors including the quality and quantity of nutrients, the individual responses to certain nutrients dietary patterns, and emergingly the timing of nutrient intake. Although several metabolic diseases are the most evident result of the pathological conditions affected by diet, diet itself may directly or indirectly, via its effects on metabolic health, modulate pathogenetic mechanisms and pathways linked with cancer and CVD [25].

The current trends in nutritional epidemiology include examining the diet-health interaction through a holistic approach, instead of focusing on isolated nutrients and food items. As such, dietary patterns capture the combination of nutrients, rather than their effects in isolation [26]. While individual dietary compounds may exert beneficial effect, even in well controlled clinical trials, it has been observed that their success and beneficial effect are not consistent. Therefore, the combination of nutrients rather than isolated nutrients, even in the case of several bioactive compounds, can be seen as the main discriminant of the effect of diet on health [26]. 
It is important to note that there are significantly less studies that focus on the effect of dietary patterns on health and disease prevention, compared with studies focused on a singular compound. This is mainly due to several factors such as practicality of the study, duration and treatments approached and near impossibility of accounting for all factors associated with individual's dietary intake [26]. Therefore, determining the individual's intake by adhering to different dietary patterns in the form of dietary index has been seen as the more approachable method of measuring and quantifying the overall dietary intake. Several tools have been developed and validated to quantify the effect of dietary patterns on human health outcomes such as; Dietary Antioxidant Index (DAI), Health Diet Indicator (HDI), Mediterranean Diet Score (MDS) and the Dietary Inflammatory Index (DII) [27,28]. The DII was developed to provide a quantitative means for assessing the role of diet in relation to health outcomes such as blood levels of inflammatory biomarkers ranging from several cytokines, IL's, and Creactive protein (CRP) to development of chronic diseases [29]. Since its development, DII has been associated with a wide range of major clinical endpoints such as development of CVD, cardiometabolic risk factors and conditions, cancer and overall mortality [30-33]. Focusing on CVD, several reports have been conducted to evaluate the associations between the anti-inflammatory potential of whole dietary patterns (index defined) and onset of cardiometabolic disorder indicating that adherence to pro-inflammatory diets constitutes a higher risk for CVD onset and mortality [34-36]. Similarly, evidence from various epidemiological studies and systematic literature reviews with meta-analyses, have proposed the consistent associations between DII and the incidence of cancer across different populations and cancer types [34-37]. A meta-analysis by Fowler and Akinyemiju [38] examined the association between the DII score and any cancer risk incidence or mortality in 24 included studies. The findings indicate that there is a consistent and significant positive association between higher DII and cancer incidence. The individuals in the highest DII category ( $v s$ lowest) had a $25 \%$ increased risk of overall cancer incidence (RR: $1.25,95 \%$ CI: $1.13-1.50)$; 75\% higher odds of cancer (OR: $1.75,95 \%$ CI: $1.43-2.16$ ) and 67\% increased risk of cancer mortality (RR: $1.67,95 \%$ CI: 1.13-2.48) [38]. In contrast, the analysis of data from cohort studies such as the ATTICA cohort, have also identified that adherence to antiinflammatory foods had a stronger association with lowering of disease incidence than the disease initiating effects of pro-inflammatory foods [33]. While this data still remains conflicting, the majority of the evidence strongly supports that anti-inflammatory foods may elicit more beneficial and protective health results rather than the avoidance of proinflammatory foods [39-41].

\subsection{Healthy dietary patterns and CVD}

Among the variety of dietary patterns that are currently gaining momentum within the scientific community, MedD has been voted as the healthiest dietary pattern and widely described for its beneficial effects on overall health, and reduction in incidence of cancer and CVD. Although there is no conclusive evidence on the potential mechanisms of action, the reduced CVD morbidity and mortality risk was proposed through several serum antioxidant and antithrombotic mechanisms or by mitigating the negative impact of conventional risk factors. To this issue, there are several meta-analyses which document either the direct positive impact of MedD or the indirect action through controlling major risk factors such as metabolic syndrome and type II diabetes (T2DM) [10,12,13,40-42].

In addition to findings from meta-analyses of cohort studies, large well-controlled clinical trials have also demonstrated the cardioprotective properties of higher adherence to MedD. The Lyon Diet Heart Study in the mid of 1990s [40], recruited participants $(n=605)$ with established coronary heart disease and followed them each year for 5 years. The findings of this study have indicated that individuals in the experimental group that consumed significantly less saturated fat, cholesterol and linoleic acid and more oleic and alpha-linoleic acid $(\mathrm{n}=302)$ had lower mortality than the control group (RR: $0.30 ; 95 \%$ CI: $0.11-0.82$; $p=0.02)$. After this, the landmark clinical trial with the acronym PREDIMED trial (Prevención con Dieta Mediterránea) in the 2010's was launched with a sample of freeof-CVD individuals at increased cardiometabolic risk [43]. The PREDIMED trial revealed about 30\% lower CVD risk in participants assigned to MedD supplemented either with extra virgin olive oil or nuts compared with a typical lowfat diet [43]. An important observation here is that despite the increase in participants' daily fat intake - which imply an increase in energy intake - the advantage of MedD over a typical low-fat diet was retained. In addition to this, participants assigned in the MedD seemed to lose more weight compared with their control counterparts in 5-year followup period [44]. Indeed, as supported by a meta-analysis of RCTs, MedD seems to be a dietary pattern with beneficial effects even in weight management — despite its high fat content [45].

In line with the existing evidence on beneficial effects of adhering to MedD, plant-based diets have also been described for their cardioprotective properties. Plant foods such as fruits and vegetables, whole-grain products, legumes as well as beverages such as coffee and tea, especially green tea, are consistently supported in the literature as yielding beneficial cardiac effects [46-50]. At the same time, these dietary patterns are also characterized by moderate to low consumption of animal foods, in particular low consumption of processed and red meats [51]. In this regard, a study by Dinu et al. [16] of pooled data from 10 different cohorts has reported a $25 \%$ lower risk for is- 
chemic heart disease incidence and/or mortality in individuals who adhered to a vegetarian diet compared to nonvegetarian. Similar observations were also reported in the EPIC-Oxford study [52] further supporting the relationship between plant-based diets and beneficial effects on the cardiovascular health outcomes.

Interestingly, the evidence from randomised controlled trials and observational studies on adherence to plant-based dietary patterns and improvements in cardiometabolic health remains relatively conflicting. Perhaps the better approach is considering a consumption of antiinflammatory foods, as chronic and systemic inflammation is a very common underlying factor associated with CVD overall [53]. The findings of the E3N cohort study by MacDonald et al. [39] reported a link between DII and hypertension. The findings of this study indicated a link between anti-inflammatory foods and a reduction in risk of CVD and hypertension development. Results also suggest that there was a stronger association between the DII and hypertension amongst healthy-lean women proposing that higher DII may have stronger influence in individuals that are perceived as healthy [39]. Similarly, in the same cohort, a study by Laouali et al. [54] attempted to assess the link between DII score and the development of T2DM. The findings of this study have indicated that the consumption of pro-inflammatory food was linked to increased incidence of developing T2DM [54].

\subsection{Healthy dietary patterns and cancer}

Diets lower in animal-based protein sources such as the MedD are proposed to therapeutically exploit the physiological [5] as well as metabolic alterations and reprograming seen in cancer cell metabolism [27]. A higher intake of protein, defined by $20 \%$ of the overall energy intake of total protein has shown an increased risk of cancer development and cancer related mortality in $75 \%$ of individuals aged 50-65 [27]. Similarly, the findings of the NHANES III longitudinal study by Levine et al. [55], conducted over 18 years and comprising of 6381 adults over the age of 50, whose diets were categorised as either high in protein $(20 \%$ protein), or low in protein (less than $10 \%$ protein) indicated that those in the high protein category, exhibited a 4-fold increased incidence of death due to cancer [55]. Nevertheless, the findings of these longitudinal cohort studies should be carefully interpreted not as the cause-and-effect relationship.

A study by Sánchez et al. [56], investigated the potential protective effect of diet in people with increased risk of developing cancer such as the individuals that partake in smoking tobacco or consuming alcohol. The findings of this study, which compared 375 participants with oral/oropharynx cancer to 375 control participants, with no current alcohol or smoking health concerns over 3 years, have indicated that there was a significant inverse relationship between risk of developing oral cancer and consump- tion of fruits and green vegetables. The authors of this study proposed that these food groups may provide potential protective properties due to the several phytochemicals (mainly polyphenols) found within the fruit and vegetables [56].

\subsection{Dietary restriction, intermittent fasting, cardiovascular disease and cancer}

The initial exploration of calorie restricted diets in epidemiological studies draw back to negative political and global events such as World War II, where food and occupation shortages, in addition to deliberate caloric restrictions were implemented in several countries. Following its cessation, epidemiological studies have reported a decrease in incidence of cardiovascular disease and some cancers [27]. These purported preventative effects to long-term adherence of a calorie restricted diet have been associated with reduction in inflammatory markers, telomerase activity and growth factors (IGF-1) associated with uncontrolled cellular proliferation in certain types of cancer [27].

Intermittent fasting diets have received an increase in popularity over the past decades particularly with respect to clinical intervention studies relating to the prevention of CVD [57-60]. Furthermore, in a relatively recent statement by the American Heart Association summarizing the effects of meal timing and frequency on CVD prevention, a whole subunit was deviated to the effect of intermittent fasting on cardiac health. In this statement, the focus was emphasised on two versions of fasting: the alternate day fasting and the periodic fasting [57-60]. In addition, a review by Tinsley and Horne [59], further supports the notion that intermittent fasting may show new research initiatives are demanded to improve the knowledge base.

The metabolic shift observed in the Warburg effect is evidently a major contributing factor to cancer progression and it is classified as one of the hallmarks of cancer development. The current evidence of different caloric restriction diets is mainly composed of studies focusing on the effect in patients undergoing radiation and/or chemotherapy with associations in caloric restrictions enhancing the effects of radiation and chemotherapy [61]. It is proposed that a calorie restricted diets target healthy and cancer cells differently. The healthy cells in this case are proposed to better conserve physiological cell signaling pathways and combat cellular stress and damage, while rendering cancer cells vulnerable to chemotherapeutic agents because of their overwhelming need to find other energy sources [61].

Intermittent fasting, such as the one performed during the Muslim faith Ramadan celebrations, have also been associated with the reduced adverse effects from chemotherapy and improvement in quality of life in cancer patients [62]. Furthermore, a study by Bauersfeld et al. [63] compared the effect of short-term fasting to adhering to MedD in women with breast and ovarian cancer. The findings of this study indicated that short-term fasting more effectively reduced the adverse reactions of chemotherapy, leading to 
improved quality of life [63]. Nevertheless, the findings in both studies reported could also be interpreted from the tolerance perspective of chemotherapy as patients may be able to better tolerate treatment and may therefore improve overall outcome.

Currently, there is still relatively limited research focusing on the overall effect of caloric restriction as a standalone agent on cancer development and progression. A study by Brandhorst et al. [64], mentions that there have been studies who observe prevention of pre-malignant lesions. Furthermore, a study by Tan-Shalaby et al. [65], investigated an altered version of the ketogenetic diets in a clinical setting for advanced solid-tumor cancer cases, not currently treated with chemotherapy agents. The findings of this study indicated that patients who reduced body weight by $10 \%$ and those who were more compliant had improved quality of life in addition to an increase in overall life expectancy. Furthermore, this type of diet was particularly effective in patients with melanoma and lung cancer [65].

The current evidence supports that caloric reduction may prevent tumorigenesis as it prevents the metabolic shift observed in the Warburg effect. The underlying mechanisms are not well understood; however, theories suggest that this shift in metabolism promotes processes like autophagy which may help to prevent tumorigenesis [59,64]. Additionally, the macronutrients that should be restricted to result in calorie restriction and at the same time have a beneficial metabolic effect is still an active research field in clinical nutrition. Starting with carbohydrates, the aggravating role of total sugars and in general simple carbohydrates like glucose and fructose is well-demonstrated [66]. Nevertheless, dietary patterns that significantly reduce total carbohydrate intake have mixed effects on cardiometabolic health; a meta-analysis suggested that such diets have beneficial effects on weight control, blood pressure and triglycerides yet not LDL-C $[67,68]$. Furthermore, the dietary restriction of specific amino acids such as glycine, serine and leucine have been proposed to potentially provide some benefits [69]. It is theorized that these amino acids contribute to metabolic dysregulation, that sees the metabolic shift to the Warburg effect, providing additional energy to proliferate and metastasize. Leucine is already identified as a pharmaceutic target in medication such as Rapamycin. Nevertheless, the current understanding is limited on how this could be exploited therapeutically with diet as it is highly dependent on the location of the tumor(s) and the host immune health [70].

\section{Can nutrigenomics offer some solutions in the hitherto epidemiological evidence}

As previously discussed, the evidence from large epidemiologic studies has demonstrated that healthy dietary patterns and restrictive diets are associated with reduced risk of CVD and cancer. However, not much is known about the mechanisms driving these associations at the molecular level. Nutrigenomics and nutri-epigenetics go beyond epidemiological and symptom-based evidence to molecularly and genetically substantiate evidence that dietary changes may modulate disease. They investigate nutrient-gene interactions, how diet influences and interacts with the genome, modulates expression of genes and thus affects the body [71]. Nutrigenomics and nutri-epigenetics are fields that are still relatively new, therefore there have been limited findings regarding the influence of dietary patterns on health on a genomic level. Most studies have been mainly investigating MedD, providing an insight into the genomic effects of a diet on CVD and cancer [71-73].

One study involving obese women on caloric restriction over 6 months, who lost over $3 \%$ of body weight, showed hypermethylation at three genomic loci in their subcutaneous adipose tissue, containing genes involved in weight control and insulin secretion [74]. Additionally, caloric restriction effect in adipose tissue on these participants also induced transcriptomic changes, modulating expression of genes involved in angiogenesis that have been linked with obesity and its complications [72,73]. These findings may further support the proposal that reduced caloric intake diets may act as modulating factors on adipocyte reprogramming and may in-turn help both prevent and treat cardiometabolic diseases including the CVD [72,73].

One study investigated epigenetic changes in adipocytes after dietary intervention, in hopes of beneficially modulating lipid compartmentalisation, and for adipocytes to utilise excessive energy more efficiently. A study by Dahlman et al. [74] observed obese female patients, who underwent bariatric surgery and were subjected to reduced caloric intake. Those with a significant reduction in body weight, defined as a reduction by around $27 \%$ showed profound epigenetic changes, including reduced global DNA methylation levels and alteration to the methylation of genes associated with T2D development and obesity [72,74]. These genes included genes linked with regulation of blood glucose levels IRS1 INSR, homeostasis CETP, LCAT, body weight LEPR, FTO, adipose tissue function mTOR and RPTOR and epigenetics FOXP2 HDAC4 and DNMT3B, many of these genes mTOR and FOXP specifically are also targets in the treatment of cancer $[72,74]$. While this study focuses on surgical means of body mass reduction, the underlying principle may still be applicable to other means of weight loss such as diet, an avenue which may be fruitful to investigate further.

Another aspect to consider within this topic is telomere length. Telomeres are repetitive DNA sequences found at the ends of the chromosomes that protect their physical integrity and shorten with each cell division [75,76]. Accelerated telomere shortening has been associated with pathological mechanisms such as inflammation, oxidative stress and genomic instability and may, therefore, influence 
CVD and cancer development. High adherence to MedD has been associated with longer telomeres in a cohort of healthy, middle-aged women [75]. Additionally, the improvements in obesity indices were observed in subjects at high cardiovascular risk whose telomere length increased after a 5-year intervention with adhering with MedD [76]. To further identify a potential genomic basis underlying MedD effect, several studies by García-Calzón and colleagues investigated the relationship between leukocyte telomere length and the genetic variants in PPAR 2 (peroxisome proliferator-activated receptor) $[72,76,77]$. Particularly three variants were analysed, the most common Pro/Pro genetype, and the Pro/Ala and Ala/Ala variants. The presence of the variant Ala allele, specifically the Pro12Ala polymorphism has been previously shown to reduce PPAR $\gamma 2$ transcriptional activity in adipose tissue and was associated with lower cardiovascular risk and higher effectiveness of lifestyle interventions [78,79]. The results of the study by García-Calzón et al. [77] showed that a 5 year-intervention with MedD reduced telomere shortening in subjects carrying the variant Ala allele of $P P A R \gamma 2$, while this effect was not observed in subjects with the more common Pro/Pro genotype. Moreover, in Ala allele carriers, better adherence to MedD was linked with higher telomere length. Thus, these studies propose that telomere shortening could be modulated by adhering to dietary patterns, highlighting the importance of the genetic makeup of an individual and its influence on the outcomes of dietary intervention.

It is proposed that MedD can also interact with polymorphisms in certain genes associated with metabolism and inflammation, thereby influencing the health outcomes, particularly regarding cardiovascular health. For example, certain polymorphisms in the TCF $7 L 2$ (transcription factor 7-like 2) gene have been associated with T2DM and could also be linked with increased lipid levels and CVD risk. Results from the PREDIMED study showed that in subjects with the highest risk TCF7L2 polymorphism, TT genotype, a great adherence to MedD led to lower fasting glucose and lipids, and also reduced stroke incidence [80]. Another study within the PREDIMED trial showed that high MedD adherence potentiated the protective effects of the rs3812316 variant in the MLXIPL (Max-like protein $\mathrm{X}$ interacting protein-like), a gene coding for carbohydrate response element binding protein, against hypertriglyceridemia and myocardial infarction [80]. Similarly, the protective effect of MedD was also observed in the ATTICA study, where adhering to this diet was associated with reduced homocysteine concentrations in individuals with TT and CT genotype for MTHFR (methylenetetrahydrofolate reductase) polymorphism $(677 \mathrm{C} \rightarrow \mathrm{T}$ mutation), which could consequently influence their coronary risk [81]. Polymorphisms in MTHFR and another gene involved in the one-carbon metabolism pathway, MTR (methionine synthase), have also been reported to influence the response of MED on cancer risk [82].

Beyond interacting with genetic polymorphisms, diet can act as an epigenetic modulator, influencing DNA methylation in certain genes or affecting global DNA methylation levels. DNA methylation is a reversible epigenetic modulation in which alterations have been associated with causing and influencing different pathogenesis, including CVD and cancer. Several studies have also reported that MedD is involved in changes in DNA methylation. For example, a study within the PREDIMED trial reported associations between MedD and methylation in TCF7L2 and FTO (alpha-ketoglutarate dependent dioxygenase) genes, closely linked to diabetes and stroke, while another study reported the link between MEdD and methylation in several inflammation-related genes (i.e., $E F F 2$, LEPR, PPARGC1B, MAPKAPK2, IFRD1) in peripheral blood cells $[83,84]$. Moreover, in healthy women, adherence to MedD was positively associated with long interspersed nuclear elements (LINE-1) methylation, a surrogate marker of global DNA methylation, which aberrant methylation was reported to associate with genomic instability, CVD and cancer risks $[73,85,86]$.

\section{Conclusions}

Healthy dietary patterns may address diseases which have a substantial link to modifiable factors, such as CVD and several different cancers. That is, healthy dietary patterns that is inclusive of anti-inflammatory foods, may better promote health than simply avoiding pro-inflammatory foods. Epidemiological, biomarker and epigenetic evidence (all in their own rights) identify that both selective and restrictive diets may help prevent the occurrence and progression of CVD and cancers, in addition to prolonging life, increasing quality of life and reducing symptoms in individuals who already live with these diseases. Furthermore, the presented evidence of the currently available studies also proposes the existence of different genomic mechanisms through which diet may exert its effects in the prevention of chronic diseases. They show the complexity of the relationship between diet and genome and highlight that some individuals will benefit more from adhering to specific dietary patterns. The results from these initial studies should encourage further research within the newly emerging field and investigation into the effects of other dietary patterns on a genomic level.

\section{Abbreviations}

CVD, Cardiovascular disease; BMI, Body Mass Index; TNF- $\alpha$, Tumour necrosis factor alpha; IL, Interleukins; DASH, Dietary Approached to Stop Hypertension; MedD, Mediterranean diet; DAI, Dietary antioxidant index; HDI, Health diet indicator; MDS, Mediterranean diet score; DII, Dietary inflammatory index; CRP, C-reactive protein; T2DM, Type 2 Diabetes Mellitus; PREDIMED, Prevención con Dieta Mediterránea. 


\section{Author contributions}

Conceptualization- $\mathrm{HB}, \quad \mathrm{MK}$ and $\mathrm{NN}$; methodology - HB, MK, IK, DS, MZ and NN; validation$\mathrm{HB}, \mathrm{MK}$, and NN; formal analysis- $\mathrm{HB}, \mathrm{MK}, \mathrm{IK}, \mathrm{DS}, \mathrm{MZ}$ and $\mathrm{NN}$; investigation- $\mathrm{HB}, \mathrm{MK}$ and $\mathrm{NN}$; resources- $\mathrm{NN}$; data curation-HB; writing - original draft preparation$\mathrm{HB}$ and $\mathrm{NN}$; writing - review and editing - $\mathrm{HB}$, and $\mathrm{NN}$; visualization- $\mathrm{HB}$ and $\mathrm{NN}$; supervision- $\mathrm{NN}$; project administration-NN. All authors have read and agreed to the published version of the manuscript.

\section{Ethics approval and consent to participate}

Not applicable.

\section{Acknowledgment}

We would like to thank Ms Amanda Bulman for her assistance in proof reading the final version of the manuscript. We would also like to thank all peer reviewers for their opinions and suggestions.

\section{Funding}

This research received no external funding.

\section{Conflict of interest}

The authors declare no conflict of interest. Matina Kouvari is serving as one of the Guest editors of this journal. We declare that Matina Kouvari had no involvement in the peer review of this article and has no access to information regarding its peer review. Full responsibility for the editorial process for this article was delegated to Samy I. McFarlane.

\section{References}

[1] D'Alessandro A, De Pergola G, Silvestris F. Mediterranean Diet and cancer risk: an open issue. International Journal of Food Sciences and Nutrition. 2016; 67: 593-605.

[2] Ellulu MS, Patimah I, Khaza'ai H, Rahmat A, Abed Y. Obesity and inflammation: the linking mechanism and the complications. Archives of Medical Science. 2017; 4: 851-863.

[3] Wilkins E, Wilson L, Wickramasinghe K, Bhatnagar P, Leal $\mathrm{J}$, Luengo-Fernandez R, et al. European Cardiovascular Disease Statistics. European Heart Network, Brussels. 2017. Available at: https://www.ehnheart.org/images/CVD-statistics-repor t-August-2017.pdf (Accessed: 10 October 2021).

[4] World Cancer Research Fund International. About the Continuous Update Project. WCRF International. 2021. https:// www.wcrf.org/diet-and-cancer/continuous-update-project (Accessed: 10 October 2021).

[5] Tosti V, Bertozzi B, Fontana L. Health Benefits of the Mediterranean Diet: Metabolic and Molecular Mechanisms. The Journals of Gerontology: Series A. 2018; 73: 318-326.

[6] Yetley EA, DeMets DL, Harlan WR. Surrogate disease markers as substitutes for chronic disease outcomes in studies of diet and chronic disease relations. The American Journal of Clinical Nutrition. 2017; 106: 1175-1189.

[7] Uramova S, Kubatka P, Dankova Z, Kapinova A, Zolakova B, Samec M, et al. Plant natural modulators in breast cancer prevention: status quo and future perspectives reinforced by predic- tive, preventive, and personalized medical approach. The EPMA Journal. 2019; 9: 403-419.

[8] Chareonrungrueangchai K, Wongkawinwoot K, Anothaisintawee T, Reutrakul S. Dietary Factors and Risks of Cardiovascular Diseases: An Umbrella Review. Nutrients. 2020; 15; 12; 4: 1088 .

[9] Rodríguez-Monforte M, Flores-Mateo G, Sánchez E. Dietary patterns and CVD: a systematic review and meta-analysis of observational studies. The British Journal of Nutrition. 2015; 114: 1341-1359.

[10] Panagiotakos D, Notara V, Kouvari M, Pitsavos C. The Mediterranean and other Dietary Patterns in Secondary Cardiovascular Disease Prevention: a Review. Current Vascular Pharmacology. 2016; 14: 442-451.

[11] Schwingshackl L, Schwedhelm C, Galbete C, Hoffmann G. Adherence to Mediterranean Diet and Risk of Cancer: an Updated Systematic Review and Meta-Analysis. Nutrients. 2017; 9: 1063.

[12] Grosso G, Marventano S, Yang J, Micek A, Pajak A, Scalfi L, et al. A comprehensive meta-analysis on evidence of Mediterranean diet and cardiovascular disease: are individual components equal? Critical Reviews in Food Science and Nutrition. 2017; 57: 3218-3232.

[13] Tang C, Wang X, Qin LQ, Dong JY. Mediterranean Diet and Mortality in People with Cardiovascular Disease: A MetaAnalysis of Prospective Cohort Studies. Nutrients. 2021; 13: 2623.

[14] Rosato V, Temple NJ, La Vecchia C, Castellan G, Tavani A, Guercio V. Mediterranean diet and cardiovascular disease: a systematic review and meta-analysis of observational studies. European Journal of Nutrition. 2019; 58: 173-191.

[15] Esposito K, Maiorino MI, Bellastella G, Chiodini P, Panagiotakos D, Giugliano D. A journey into a Mediterranean diet and type 2 diabetes: a systematic review with meta-analyses. BMJ Open. 2015; 5: e008222.

[16] Dinu M, Abbate R, Gensini GF, Casini A, Sofi F. Vegetarian, vegan diets and multiple health outcomes: a systematic review with meta-analysis of observational studies. Critical Reviews in Food Science and Nutrition. 2017; 57: 3640-3649.

[17] Weihrauch-Blüher S, Schwarz P, Klusmann J. Childhood obesity: increased risk for cardiometabolic disease and cancer in adulthood. Metabolism: Clinical and Experimental. 2019; 92: $147-152$.

[18] Shamriz O, Leiba M, Levine H, Derazne E, Keinan-Boker L, Kark JD. Higher body mass index in 16-19 year-old Jewish Adolescents of North African, Middle Eastern and European Origins is a Predictor of Acute Myeloid Leukemia: a cohort of 2.3 million Israelis. Cancer Causes \& Control. 2017; 28; 331-339

[19] Leiba M, Leiba A, Keinan-Boker L, Avigdor A, Derazne E, Levine $\mathrm{H}$, et al. Adolescent weight and height are predictors of specific non-Hodgkin lymphoma subtypes among a cohort of 2,352,988 individuals aged 16 to 19 years. Cancer. 2016; 122: $1068-1077$.

[20] Friedman D, Tonorezos E, Cohen P. Diabetes and Metabolic Syndrome in Survivors of Childhood Cancer. Hormone Research in Paediatrics. 2019; 91: 118-127.

[21] Charles-Messance H, Mitchelson KAJ, De Marco Castro E, Sheedy FJ, Roche HM. Regulating metabolic inflammation by nutritional modulation. Journal of Allergy and Clinical Immunology. 2020; 146: 706-720.

[22] Hanahan D, Weinberg RA. Hallmarks of cancer: the next generation. Cell. 2011; 144: 646-674.

[23] Kuspriyanti N, Ariyanto E, Syamsunarno M. Role of Warburg Effect in Cardiovascular Diseases: A Potential Treatment Option. The Open Cardiovascular Medicine Journal. 2021; 1: 6 17.

[24] Nakagawa T, Lanaspa MA, Millan IS, Fini M, Rivard CJ, 
Sanchez-Lozada LG, et al. Fructose contributes to the Warburg effect for cancer growth. Cancer Metabolism. 2020; 8; 16.

[25] Heidemann C, Schulze MB, Franco OH, van Dam RM, Mantzoros CS, Hu FB. Dietary patterns and risk of mortality from cardiovascular disease, cancer, and all causes in a prospective cohort of women. Circulation. 2008; 118: 230-237.

[26] Liu RH. Potential Synergy of Phytochemicals in Cancer Prevention: Mechanism of Action. The Journal of Nutrition. 2004; 134 : 3479S-3485S.

[27] Hanjani NA, Vafa M. Protein Restriction, Epigenetic Diet, Intermittent Fasting as New Approaches for Preventing Age-associated Diseases. International Journal of Preventive Medicine. 2018; 9: 58.

[28] Gil Á, Martinez de Victoria E, Olza J. Indicators for the evaluation of diet quality. Nutricion Hospitalaria. 2015; 3: 128-144.

[29] Li D, Hao X, Li J, Wu Z, Chen S, Lin J, et al. Dose-response relation between dietary inflammatory index and human cancer risk: evidence from 44 epidemiologic studies involving 1,082,092 participants. The American Journal of Clinical Nutrition. 2021; 107: 371-388.

[30] Ji M, Hong X, Chen M, Chen T, Wang J, Zhang N. Dietary inflammatory index and cardiovascular risk and mortality: A meta-analysis of cohort studies. Medicine. 2020; 99: e20303.

[31] Kouvari M, Panagiotakos DB, Naumovski N, Chrysohoou C, Georgousopoulou EN, Yannakoulia M, et al. Dietary antiinflammatory index, metabolic syndrome and transition in metabolic status; a gender-specific analysis of ATTICA prospective study. Diabetes Research and Clinical Practice. 2020; 161: 108031.

[32] Kaluza J, Håkansson N, Harris HR, Orsini N, Michaëlsson K, Wolk A. Influence of anti-inflammatory diet and smoking on mortality and survival in men and women: two prospective cohort studies. Journal of Internal Medicine. 2019; 285: 75-91.

[33] Jayedi A, Emadi A, Shab-Bidar S. Dietary Inflammatory Index and Site-Specific Cancer Risk: A Systematic Review and Dose-Response Meta-Analysis. Advances in Nutrition. 2018; 9; 4: $388-403$.

[34] Shivappa N, Godos J, Hébert JR, Wirth MD, Piuri G, Speciani $\mathrm{AF}$, et al. Dietary Inflammatory Index and Cardiovascular Risk and Mortality-A Meta-Analysis. Nutrients. 2018; 10: 200.

[35] Namazi N, Larijani B, Azadbakht L. Dietary Inflammatory Index and its Association with the Risk of Cardiovascular Diseases, Metabolic Syndrome, and Mortality: a Systematic Review and Meta-Analysis. Hormone and Metabolic Research. 2018; 50: 345-358.

[36] Ruiz-Canela M, Zazpe I, Shivappa N, Hébert JR, Sánchez-Tainta $\mathrm{A}$, Corella D, et al. Dietary inflammatory index and anthropometric measures of obesity in a population sample at high cardiovascular risk from the PREDIMED (PREvención con DIeta MEDiterránea) trial. The British Journal of Nutrition. 2015; 113 : 984-995.

[37] Ostan R, Lanzarini C, Pini E, Scurti M, Vianello D, Bertarelli $\mathrm{C}$, et al. Inflammaging and cancer: a challenge for the Mediterranean diet. Nutrients. 201T; 7: 2589-2621.

[38] Fowler ME, Akinyemiju TF. Meta-analysis of the association between dietary inflammatory index (DII) and cancer outcomes. International Journal of Cancer. 2017; 141: 2215-2227.

[39] MacDonald CJ, Laouali B, Madika A-L, Mancini F, BoutronRuault M-C. Dietary inflammatory index, risk of incident hypertension, and effect modification from BMI. Nutrition Journal. $2020 ; 19 ; 62$

[40] de Lorgeril M, Renaud S, Salen P, Monjaud I, Mamelle N, Martin JL, et al. Mediterranean alpha-linolenic acid-rich diet in secondary prevention of coronary heart disease. The Lancet. 1994; 343: 1454-1459.

[41] Koloverou E, Esposito K, Giugliano D, Panagiotakos D. The effect of Mediterranean diet on the development of type 2 diabetes mellitus: a meta-analysis of 10 prospective studies and 136,846 participants. Metabolism: Clinical and Experimental. 2014; 63: 903-911.

[42] Kastorini C, Milionis HJ, Esposito K, Giugliano D, Goudevenos JA, Panagiotakos DB. The effect of Mediterranean diet on metabolic syndrome and its components: a meta-analysis of 50 studies and 534,906 individuals. Journal of the American College of Cardiology. 2011; 57: 1299-1313.

[43] Ros E, Martínez-González MA, Estruch R, Salas-Salvadó J, Fitó M, Martínez JA, et al. Mediterranean Diet and Cardiovascular Health: Teachings of the PREDIMED Study. Advances in Nutrition. 2014; 5: 330S-336S.

[44] Estruch R, Martínez-González MA, Corella D, Salas-Salvadó J, Fitó M, Chiva-Blanch G, et al. Effect of a high-fat Mediterranean diet on bodyweight and waist circumference: a prespecified secondary outcomes analysis of the PREDIMED randomised controlled trial. The Lancet Diabetes \& Endocrinology. 2019; 7: e6-e17.

[45] Esposito K, Kastorini C, Panagiotakos DB, Giugliano D. Mediterranean diet and weight loss: meta-analysis of randomized controlled trials. Metabolic Syndrome and Related Disorders. 2011; 9: 1-12.

[46] Aune D, Giovannucci E, Boffetta P, Fadnes LT, Keum N, Norat $\mathrm{T}$, et al. Fruit and vegetable intake and the risk of cardiovascular disease, total cancer and all-cause mortality-a systematic review and dose-response meta-analysis of prospective studies. International Journal of Epidemiology. 2017; 46: 1029-1056.

[47] Zong G, Gao A, Hu FB, Sun Q. Whole Grain Intake and Mortality from all Causes, Cardiovascular Disease, and Cancer. Circulation. 2016; 133: 2370-2380.

[48] Bechthold A, Boeing H, Schwedhelm C, Hoffmann G, Knüppel $\mathrm{S}$, Iqbal $\mathrm{K}$, et al. Food groups and risk of coronary heart disease, stroke and heart failure: a systematic review and dose-response meta-analysis of prospective studies. Critical Reviews in Food Science and Nutrition. 2017; 59: 1071-1090.

[49] Ding M, Bhupathiraju SN, Satija A, van Dam RM, Hu FB. LongTerm Coffee Consumption and Risk of Cardiovascular Disease. Circulation. 2014; 129: 643-659.

[50] Chung M, Zhao N, Wang D, Shams-White M, Karlsen M, Cassidy A, et al. Dose-Response Relation between Tea Consumption and Risk of Cardiovascular Disease and all-Cause Mortality: a Systematic Review and Meta-Analysis of PopulationBased Studies. Advances in Nutrition. 2020; 11: 790-814.

[51] Galbete C, Kröger J, Jannasch F, Iqbal K, Schwingshackl L, Schwedhelm C, et al. Nordic diet, Mediterranean diet, and the risk of chronic diseases: the EPIC-Potsdam study. BMC Medicine. 2018; 16: 99.

[52] Tong T, Appleby P, Bradbury K, Perez-Cornago A, Travis R, Clarke R, et al. Risks of ischaemic heart disease and stroke in meat eaters, fish eaters, and vegetarians over 18 years of followup: results from the prospective EPIC-Oxford study. British Medical Journal 2019; 366: 14897.

[53] Kwok CS, Umar S, Myint PK, Mamas MA, Loke YK. Vegetarian diet, Seventh Day Adventists and risk of cardiovascular mortality: a systematic review and meta-analysis. International Journal of Cardiology. 2014; 176: 680-686.

[54] Laouali N, Mancini FR, Hajji-Louati M, El Fatouhi D, Balkau B, Boutron-Ruault M, et al. Dietary inflammatory index and type 2 diabetes risk in a prospective cohort of 70,991 women followed for 20 years: the mediating role of BMI. Diabetologia. 2019; 62: 2222-2232.

[55] Levine ME, Suarez JA, Brandhorst S, Balasubramanian P, Cheng C, Madia F, et al. Low protein intake is associated with a major reduction in IGF-1, cancer, and overall mortality in the 65 and younger but not older population. Cell Metabolism. 2014; 19: 407-417.

[56] Sánchez MJ, Martínez C, Nieto A, Castellsagué X, Quintana MJ, Bosch FX, et al. Oral and oropharyngeal cancer in Spain: influ- 
ence of dietary patterns. European Journal of Cancer Prevention. 2003; 12: 49-56.

[57] Mattson MP, Longo VD, Harvie M. Impact of intermittent fasting on health and disease processes. Ageing Research Reviews. 2017; 39: 46-58.

[58] Trepanowski JF, Kroeger CM, Barnosky A, Klempel MC, Bhutani S, Hoddy KK, et al. Effect of Alternate-Day Fasting on Weight Loss, Weight Maintenance, and Cardioprotection among Metabolically Healthy Obese Adults: a Randomized Clinical Trial. JAMA Internal Medicine. 2017; 177: 930-938.

[59] Tinsley GM, Horne BD. Intermittent fasting and cardiovascular disease: current evidence and unresolved questions. Future Cardiology. 2018; 14: 47-54.

[60] St-Onge M, Ard J, Baskin ML, Chiuve SE, Johnson HM, KrisEtherton $\mathrm{P}$, et al. Meal Timing and Frequency: Implications for Cardiovascular Disease Prevention: a Scientific Statement from the American Heart Association. Circulation. 2017; 135: e96e121.

[61] Ibrahim EM, Al-Foheidi MH, Al-Mansour MM. Energy and caloric restriction, and fasting and cancer: a narrative review. Supportive Care in Cancer. 2021; 29: 2299-2304.

[62] Badar T. Safety and Feasibility of Muslim Fasting While Receiving Chemotherapy. IOSR Journal of Pharmacy. 2014; 04: 15-20.

[63] Bauersfeld SP, Kessler CS, Wischnewsky M, Jaensch A, Steckhan N, Stange R, et al. The effects of short-term fasting on quality of life and tolerance to chemotherapy in patients with breast and ovarian cancer: a randomized cross-over pilot study. BMC Cancer. 2018; 18: 476.

[64] Brandhorst S, Longo VD. Fasting and Caloric Restriction in Cancer Prevention and Treatment. Recent Results in Cancer Research. 2016; 207: 241-266

[65] Tan-Shalaby JL, Carrick J, Edinger K, Genovese D, Liman AD, Passero VA, et al. Modified Atkins diet in advanced malignancies - final results of a safety and feasibility trial within the Veterans Affairs Pittsburgh Healthcare System. Nutrition \& Metabolism. 2016; 13: 52.

[66] Khan TA, Tayyiba M, Agarwal A, Mejia SB, de Souza RJ, Wolever TMS, et al. Relation of Total Sugars, Sucrose, Fructose, and Added Sugars with the Risk of Cardiovascular Disease: A Systematic Review and Dose-Response Meta-analysis of Prospective Cohort Studies. Mayo Clinic Proceedings. 2019; 94: 2399-2414

[67] Dong T, Guo M, Zhang P, Sun G, Chen B. The effects of low-carbohydrate diets on cardiovascular risk factors: A metaanalysis. PLoS ONE. 2020; 15; 1: e0225348.

[68] Mansoor N, Vinknes KJ, Veierød MB, Retterstøl K. Effects of low-carbohydrate diets v. low-fat diets on body weight and cardiovascular risk factors: a meta-analysis of randomised controlled trials. The British Journal of Nutrition. 2016; 115: 466479.

[69] Kang J. Dietary restriction of amino acids for Cancer therapy. Nutrition \& Metabolism. 2020; 17: 20.

[70] Butler M, van der Meer LT, van Leeuwen FN. Amino Acid Depletion Therapies: Starving Cancer Cells to Death. Trends in Endocrinology \& Metabolism. 2021; 32: 367-381.

[71] Nasir A, Bullo MMH, Ahmed Z, Imtiaz A, Yaqoob E, Jadoon M, et al. Nutrigenomics: Epigenetics and cancer prevention: a comprehensive review. Critical Reviews in Food Science and Nutrition. 2019; 60: 1375-1387.

[72] Asif S, Morrow N, Mulvihill E, Kim K. Understanding Dietary Intervention-Mediated Epigenetic Modifications in Metabolic Diseases. Frontiers in Genetics. 2020; 11: 590369.

[73] Bouchard L, Rabasa-Lhoret R, Faraj M, Lavoie M, Mill J, Pérusse L, et al. Differential epigenomic and transcriptomic responses in subcutaneous adipose tissue between low and high responders to caloric restriction. The American Journal of Clinical Nutrition. 2010; 91: 309-320.

[74] Dahlman I, Sinha I, Gao H, Brodin D, Thorell A, Rydén M, et al. The fat cell epigenetic signature in post-obese women is characterized by global hypomethylation and differential DNA methylation of adipogenesis genes. International Journal of Obesity. 2015; 39: 910-919.

[75] Crous-Bou M, Fung TT, Prescott J, Julin B, Du M, Sun Q, et al. Mediterranean diet and telomere length in Nurses' Health Study: population based cohort study. British Medical Journal. 2014; 349: g6674.

[76] García-Calzón S, Gea A, Razquin C, Corella D, LamuelaRaventós RM, Martínez JA, et al. Longitudinal association of telomere length and obesity indices in an intervention study with a Mediterranean diet: the PREDIMED-NAVARRA trial. International Journal of Obesity. 2014; 38: 177-182.

[77] García-Calzón S, Martínez-González MA, Razquin C, Corella D, Salas-Salvadó J, Martínez JA, et al. Pro12Ala polymorphism of the PPAR $\gamma 2$ gene interacts with a mediterranean diet to prevent telomere shortening in the PREDIMED-NAVARRA randomized trial. Circulation. Cardiovascular Genetics. 2015; 8: 91-99.

[78] Regieli JJ, Jukema JW, Doevendans PA, Zwinderman AH, van der Graaf Y, Kastelein JJ, et al. PPAR $\gamma$ Variant Influences Angiographic Outcome and 10-Year Cardiovascular Risk in Male Symptomatic Coronary Artery Disease Patients. Diabetes Care. 2009; 32: 839-844.

[79] Rittig K, Thamer C, Machicao F, Rietig R, Stefan N, Fritsche A, et al. The Pro12Ala polymorphism in PPARG2 increases the effectiveness of primary prevention of cardiovascular disease by a lifestyle intervention. Diabetologia. 2007; 50: 1345-1347.

[80] Ortega-Azorín C, Sorlí JV, Estruch R, Asensio EM, Coltell O, González JI, et al. Amino acid change in the carbohydrate response element binding protein is associated with lower triglycerides and myocardial infarction incidence depending on level of adherence to the Mediterranean diet in the PREDIMED trial. Circulation. Cardiovascular Genetics. 2014; 7: 49-58.

[81] Dedoussis GV, Panagiotakos DB, Chrysohoou C, Pitsavos C, Zampelas A, Choumerianou D, et al. Effect of interaction between adherence to a Mediterranean diet and the methylenetetrahydrofolate reductase $677 \mathrm{C} \rightarrow \mathrm{T}$ mutation on homocysteine concentrations in healthy adults: the ATTICA Study. The American Journal of Clinical Nutrition. 2004; 80: 849-854.

[82] Kakkoura MG, Demetriou CA, Loizidou MA, Loucaides G, Neophytou I, Marcou Y, et al. Single-nucleotide polymorphisms in one-carbon metabolism genes, Mediterranean diet and breast cancer risk: a case-control study in the Greek-Cypriot female population. Genes \& Nutrition. 2015; 10: 453.

[83] Corella D, Ordovas J, Sorli J, Asensio E, Ortega C, Carrasco P, et al. Effect of the Mediterranean Diet on DNA Methylation of Selected Genes in the PREDIMED-Valencia Intervention Trial. The FASEB Journal. 2015; 29: LB242.

[84] Arpón A, Riezu-Boj JI, Milagro FI, Marti A, Razquin C, Martínez-González MA, et al. Adherence to Mediterranean diet is associated with methylation changes in inflammation-related genes in peripheral blood cells. Journal of Physiology and Biochemistry. 2016; 73: 445-455.

[85] Barchitta M, Maugeri A, La Rosa M, Agrifoglio O, La Mastra C, Agodi A. Adherence to the mediterranean diet is positively associated with LINE-1 methylation levels. European Journal of Public Health. 2018; 28: 4.

[86] Barchitta M, Maugeri A, Quattrocchi A, Barone G, Mazzoleni P, Catalfo A, et al. Mediterranean Diet and Particulate Matter Exposure Are Associated With LINE-1 Methylation: Results From a Cross-Sectional Study in Women. Frontiers in Genetics. 2018; 9: 514. 